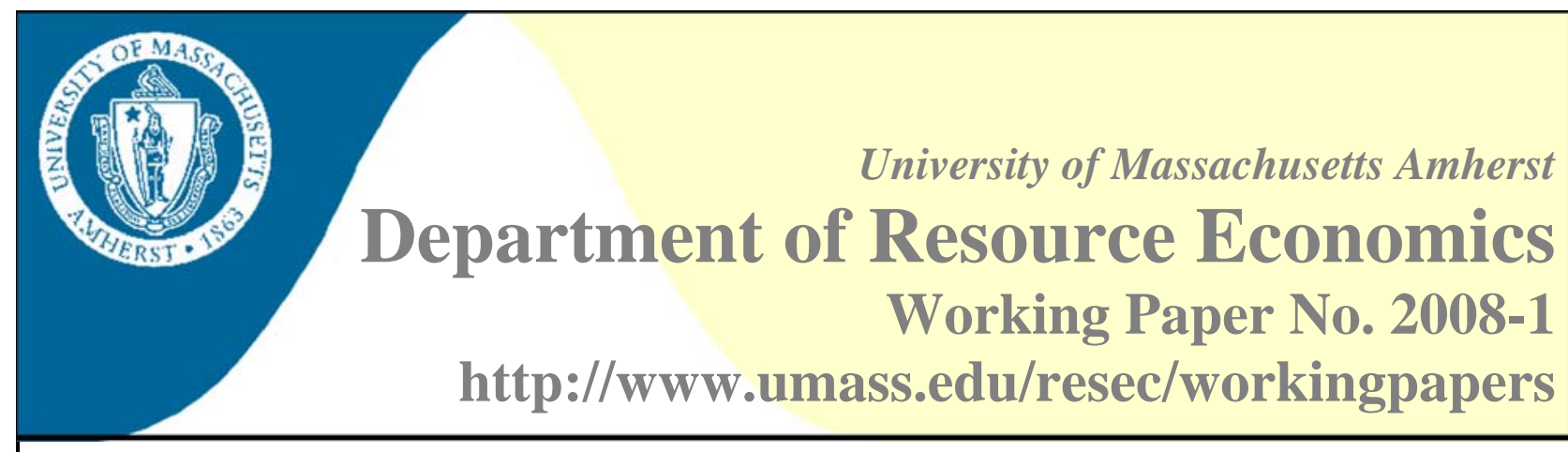

\title{
Imperfect Enforcement of Emissions Trading and Industry Welfare: A Laboratory Investigation
}

\author{
John K. Stranlund ${ }^{1}$, James J. Murphy ${ }^{2}$, and John M. Spraggon ${ }^{3}$
}

Abstract:

This paper uses laboratory experiments to investigate the performance of emission permit markets when compliance is imperfectly enforced. In particular we examine deviations in observed aggregate payoffs and expected penalties from those derived from a model of riskneutral payoff-maximizing firms. We find that the experimental emissions markets were reasonably efficient at allocating individual emission control choices despite imperfect enforcement and significant noncompliance. However, violations and expected penalties were lower than predicted when these are predicted to be high, but were about the same as predicted values when these values were predicted to be low. Thus, although a standard model of compliance with emissions trading programs tends to predict significantly higher violations than we observe when subjects have strong incentives to violate their emissions permits, individual emissions control responsibilities are distributed among firms as predicted.

Keywords: enforcement, compliance, emissions trading, permit markets, pollution, laboratory experiments

JEL Classification: C91, L51, Q58

\footnotetext{
${ }^{1}$ John K. Stranlund, Department of Resource Economics

University of Massachusetts, 214 Stockbridge Hall

80 Campus Center Way, Amherst, MA 01003-9246

E: stranlund@resecon.umass.edu P: 413-545-6328 F: 413-545-5853

${ }^{2}$ James Murphy, Department of Economics

University of Alaska Anchorage, 201-D Rasmuson Hall

3211 Providence Drive, Anchorage, AK 99508

E: murphy@uaa.alaska.edu P: 907-786-1936 F: 907-786-4115

${ }^{3}$ John M. Spraggon, Department of Resource Economics

University of Massachusetts, 212A Stockbridge Hall

80 Campus Center Way, Amherst, MA 01003-9246

E: jmspragg@resecon.umass.edu P: 413-545-6651 F: 413-545-5853
} 
March 2008

\title{
Imperfect Enforcement of Emissions Trading and Industry Welfare: A Laboratory Investigation
}

\author{
John K. Stranlund \\ Department of Resource Economics \\ University of Massachusetts Amherst \\ James J. Murphy* \\ Department of Economics \\ University of Alaska Anchorage \\ John M. Spraggon \\ Department of Resource Economics \\ University of Massachusetts Amherst
}

Acknowledgements: Primary funding for this research was provided by the U.S. EPA - Science to Achieve Results (STAR) Program grant \#R829608. Additional support was provided by the Cooperative State Research Extension, Education Service, U. S. Department of Agriculture, Massachusetts Agricultural Experiment Station, and the Department of Resource Economics under Project No. MAS00871, and by the Center for Public Policy and Administration, University of Massachusetts-Amherst. Maria Alejandra Velez, Carrie Puglisi and Maria Claudia Lopez provided outstanding research assistance. Glenn Caffery programmed the software for this project. Wendy Varner provided valuable administrative support. The authors take full responsibility for any errors or omissions. The authors acknowledge helpful comments by participants in the Seminar in Environmental Economics and Policy at Harvard University, and at Advanced Workshop in Regulation and Competition, 20th Annual Western Conference, June 2007.

\footnotetext{
* Correspondence to: James J. Murphy. Dept of Economics, Rasmuson Hall, 3211 Providence Drive, Anchorage, AK 99508. Phone: 907-786-1936. Fax: 907-786-4115. Email: jmurphy@cbpp.uaa.alaska.edu.
} 


\title{
Imperfect Enforcement of Emissions Trading and Industry Welfare: A Laboratory Investigation
}

\begin{abstract}
This paper uses laboratory experiments to investigate the performance of emission permit markets when compliance is imperfectly enforced. In particular we examine deviations in observed aggregate payoffs and expected penalties from those derived from a model of riskneutral payoff-maximizing firms. We find that the experimental emissions markets were reasonably efficient at allocating individual emission control choices despite imperfect enforcement and significant noncompliance. However, violations and expected penalties were lower than predicted when these are predicted to be high, but were about the same as predicted values when these values were predicted to be low. Thus, although a standard model of compliance with emissions trading programs tends to predict significantly higher violations than we observe when subjects have strong incentives to violate their emissions permits, individual emissions control responsibilities are distributed among firms as predicted.
\end{abstract}

Keywords: enforcement, compliance, emissions trading, permit markets, pollution, laboratory experiments

JEL Codes: C91, L51, Q58

\section{Introduction}

Much of what we understand about the advantages of emissions trading programs, particularly vis-à-vis traditional command and control approaches, is based on models of full compliance with these policies. However, emissions trading policies are not likely to perform as expected if these programs are not enforced well. Recognizing this concern, there is now a significant body of theoretical literature on the consequences of imperfect enforcement of emissions trading programs (e.g. Keeler 1991, Malik 1990 and 1992, Stranlund and Dhanda 1999, Stranlund 2007).

While the theory of the compliance and enforcement problem in emissions trading has advanced quite far, there are few empirical investigations of compliance behavior and the performance of emissions markets under imperfect compliance. The dearth of empirical research in this area is due to the fact that opportunities for empirical analyses of imperfect enforcement 
in existing trading programs are limited. ${ }^{1}$ In situations in which naturally occurring data are limited, laboratory experiments are particularly valuable. In fact, several recent papers use experiments to examine various aspects of imperfect compliance in emission trading programs (Cason and Gangadharan 2006, Murphy and Stranlund 2006 and 2007). These works provide valuable results about the compliance behavior of subjects in experimental emissions permit markets, but none deal directly with the effects of noncompliance on the social costs and benefits of these programs. ${ }^{2}$ In this study, we use the data from a series of emissions trading experiments to examine the impacts of imperfect enforcement on industry welfare. By imperfect enforcement we mean that enforcement efforts—monitoring and penalties—are not sufficient to induce full compliance by all firms.

The main value of competitive emissions permit markets is that they will produce the allocation of emissions control among firms that maximizes industry profit, given the reduction of aggregate emissions. Malik (1990) demonstrates that this result holds under reasonable circumstances even when enforcement cannot induce full compliance. Moreover, he shows that this result is independent of firms' risk preferences. Since permit markets are predicted to distribute control responsibilities efficiently, the main conceptual effect of imperfect enforcement is that individual firms will violate their permits and therefore aggregate emissions will exceed the number of permits that are put into circulation. When this occurs, it is easy to demonstrate that trading will distribute permits such that the individual violation choices of risk-

\footnotetext{
${ }^{1}$ For example, the EPA's $\mathrm{SO}_{2}$ Allowance Trading Program and the $\mathrm{NO}_{\mathrm{x}}$ Budget Program have achieved such high rates of compliance that there is simply not enough variation to conduct meaningful econometric analyses of compliance decisions in these programs. See the most recent compliance reports for these programs, U.S. EPA (2007a and 2007b).

${ }^{2}$ Although experimental techniques have been used to evaluate other policy initiatives, including some aspects of emissions trading programs (see the review by Muller and Mestelman 1998 and papers in the book edited by Isaac and Holt 1999), these techniques have not yet been widely applied to issues of regulatory enforcement. By far, the bulk of experimental analyses of compliance and enforcement is in the area of income tax compliance. See Alm and McKee (1998) and Torgler (2002) for comprehensive surveys of this literature.
} 
neutral firms will minimize aggregate expected penalties for the given level of emissions. Since the distribution of individual emissions maximizes gross profit (penalties excluded) and the distribution of individual violations minimizes expected penalties, a competitive emission trading program that is imperfectly enforced will maximize aggregate expected net profits (aggregate gross profits minus aggregate expected penalties), given the level of aggregate emissions that is achieved.

Our laboratory experiments were designed to investigate whether and how aggregate gross profit, aggregate expected penalties, and aggregate expected net profits deviate from theoretical predictions that are based on risk-neutral profit-maximizing firms when enforcement is not expected to induce their full compliance. Empirically, deviations from predictions about industry welfare can be decomposed into two types of effects. The first are individual allocation effects that account for deviations in aggregate profits and expected penalties from model predictions, given the observed levels of aggregate emissions and aggregate violations. Isolating these effects allows us to examine whether the market allocates individual emissions control responsibilities among firms so as to maximize aggregate gross profit and allocates violations to minimize aggregate expected penalties, even if observed aggregate emissions and violations differ from predicted values. It is important to note that if the distribution of individual violations does not maximize aggregate gross profits, then we will reject Malik’s assertion that emission markets will distribute individual control responsibilities efficiently even when these markets are imperfectly enforced. However, we cannot say that a permit market fails if we find that individual violation choices do not minimize aggregate expected penalties. Deviations in individual violations from predicted values that lead to higher expected penalties can be due to differences in risk preferences (or other compliance-related preferences) that are assumed away 
by the model of risk-neutral profit-maximizing behavior. The second part of deviations in gross profits and expected penalties from predictions are aggregate compliance effects that can arise if aggregate emissions and violations differ from predicted values. A difference between observed and predicted aggregate emissions and violations also do not imply a permit market failure because they can also stem from a failure of a standard model of firms to accurately predict compliance choices.

The deviations in aggregate gross profits, expected penalties, and expected net profits from model predictions, and our decompositions of these deviations into allocation and compliance effects, yield several results. One of the most important is that the experimental markets tended to allocate individual emissions control so that aggregate gross profits were very close to maximized values, given the observed levels of aggregate emissions. Thus, we find strong support for Malik's hypothesis that emissions markets will distribute individual abatement efforts efficiently despite imperfect enforcement and significant noncompliance.

However, aggregate violations were significantly lower than predicted when predicted violations were very high, while the subjects' violations were quite close to predicted values when predicted violations were lower. In all cases, individual violation choices did not minimize aggregate expected penalties at the observed levels of aggregate violations. However, because the subjects did not violate their permits as much as predicted when their violations were predicted to be high, aggregate expected penalties were significantly lower than predicted in these treatments. In the treatments in which predicted violations were lower, aggregate expected penalties were usually not statistically different from predicted values.

These results point to a failure of the model of risk-neutral profit-maximizing behavior to accurately predict compliance behavior when the subjects had very strong incentives to violate 
their permits. Despite this our observations of aggregate expected net profits—gross profits minus expected penalties — were often not very far away from our predictions. Observed aggregate expected net profits were at least $96 \%$ of their maximums for the treatments in which we predicted low violations, and between $87 \%$ and $96 \%$ of maximums for the treatments for which predicted violations were high.

With imperfectly enforced emissions trading programs the two most important items to be concerned about are the aggregate level of emissions control and the distribution of individual emissions control. Our results suggest that emissions trading is a reasonably efficient way to allocate individual emissions control responsibilities, even when enforcement is imperfect. Moreover, poorly enforced programs may not result in as much noncompliance as a standard model would predict. Depending on the benefits of pollution control in a particular setting, it is easy to imagine how lower-than-predicted violations and emissions could result in higher-thanpredicted social welfare if the additional benefit of pollution control outweighs the reduction in the expected net profits of pollution sources. This, of course, is not a justification for designing poorly enforced trading programs. Our analysis merely suggests that imperfect enforcement may not always be as costly as standard models predict.

\section{Firm behavior and market equilibrium under imperfectly enforced emission trading}

In this section we present a standard model of firm behavior and the equilibrium of an imperfectly enforced emissions trading policy that provides the foundation for our experimental design. Because all of the results in this section can be gleaned from the existing literature (in particular, Malik 1992, Stranlund and Dhanda 1999, and Stranlund 2007), we only sketch the theory. 
Consider a fixed set of heterogeneous risk neutral firms. Firm $i$ 's gross profit from emitting $q_{i}$ is given by the strictly concave gross profit function $b_{i}\left(q_{i}\right) .{ }^{3}$ Absent a regulatory motivation to reduce its emissions, the firm emits $\bar{q}_{i}$, the solution to $b_{i}^{\prime}\left(q_{i}\right)=0$. A market for emission permits will generate a permit price that motivates the firm to emit $q_{i}<\bar{q}_{i}$. For these levels of emissions, $b_{i}^{\prime}\left(q_{i}\right)>0$.

A total of $L<\sum \bar{q}_{i}$ emissions permits are distributed to the firms free of charge. Firm $i$ 's initial allocation is $l_{i}^{0}$, and it chooses to hold $l_{i}$ permits after trading is completed. Each permit confers the legal right to emit one unit. Assume competitive behavior in the permit market so that all trades take place at a constant price $p$. The analysis throughout is static.

If a firm is noncompliant, then its emissions exceed the number of permits it holds and the magnitude of its violation is $v_{i}=q_{i}-l_{i}>0$. If the firm is compliant, $q_{i}-l_{i} \leq 0$ and $v_{i}=0$. To check for compliance, each firm is audited with a known and fixed probability $\pi$. A firm that is found to be in violation is penalized according to a penalty function, $f\left(v_{i}\right)$, which is increasing and strictly convex for $v_{i} \geq 0$. The audit probability and the penalty function are exogenous and do not vary across firms.

Assuming throughout that each firm chooses positive emissions and holds a positive number of permits, firm $i$ 's objective is:

$$
\begin{aligned}
& \max _{q_{i}, l_{i}} b_{i}\left(q_{i}\right)-p\left(l_{i}-l_{i}^{0}\right)-\pi f\left(q_{i}-l_{i}\right) \\
& \text { subject to } q_{i}-l_{i} \geq 0 .
\end{aligned}
$$

\footnotetext{
${ }^{3}$ Strictly speaking, $b_{i}\left(q_{i}\right)$ is the firm's gross profit assuming that it makes all of its input and output choices optimally. See Montgomery (1972) for a demonstration of the concavity of profit in emissions for firms that are price-takers in input and output markets. Many authors choose to model firms' abatement costs rather than profits from emissions. We presented our subjects with profit functions so we use them here to maintain consistency. There is no loss of generality in our approach.
} 
Restricting the firm to $v_{i}=q_{i}-l_{i} \geq 0$ follows from the fact that a firm will never have an incentive to be over-compliant. Letting $\mathcal{L}$ denote the Lagrange equation for [1] and $\lambda_{i}$ denote the multiplier attached to the constraint $q_{i}-l_{i} \geq 0$, the first-order conditions for a solution to [1] are:

$$
\begin{aligned}
& \mathcal{L}_{q}=b_{i}^{\prime}\left(q_{i}\right)-\pi f^{\prime}\left(q_{i}-l_{i}\right)+\lambda_{i}=0 \\
& \mathcal{L}_{l}=-p+\pi f^{\prime}\left(q_{i}-l_{i}\right)-\lambda_{i}=0 \\
& \mathcal{L}_{\lambda}=q_{i}-l_{i} \geq 0, \lambda_{i} \geq 0, \lambda_{i}\left(q_{i}-l_{i}\right)=0 .
\end{aligned}
$$

Because the constraint $q_{i}-l_{i} \geq 0$ is linear and the firm's objective is strictly concave these conditions are necessary and sufficient to identify unique optimal choices of emissions, permit demand, and violation level.

As noted in the introduction, Malik (1990) demonstrated an interesting and important result concerning the performance of emission trading programs and imperfect enforcement. Under reasonable specifications of the probability of monitoring firms, which include random monitoring as in this paper, a competitive permit market will distribute individual emissions control responsibilities so that, regardless of the level of aggregate abatement actually achieved, aggregate abatement costs are minimized. Moreover, Malik showed that this result does not depend on the firms' risk preferences. Malik’s result is fully equivalent to saying that aggregate gross profit will be maximized given the actual level of aggregate emissions despite imperfect enforcement and significant noncompliance.

To demonstrate this, combine equations [2] and [3] to obtain $p=b_{i}^{\prime}\left(q_{i}\right)$, which is the familiar rule that competitive firms will choose their emissions to equate the going permit price to their marginal benefits of increased emissions. Equating marginal gross profits across firms 
gives us the necessary conditions for maximizing industry gross profit given some level of aggregate emissions. Therefore, letting $Q$ denote aggregate emissions, in a permit market equilibrium we have:

$$
p=B^{\prime}(Q) \text {, where } B(Q)=\max _{q_{i}} \sum_{i=1}^{n} b_{i}\left(q_{i}\right) \text { s.t. } \sum_{i=1}^{n} q_{i}=Q \text {. }
$$

Note that a firm's emissions choice is independent of the enforcement strategy it faces, and thus holds regardless of its compliance choice. Therefore, the ability of the permit market to allocate individual emissions choices efficiently is not affected by the enforcement strategy that is applied to the market or the risk preferences of firms. Testing this conclusion is one of the primary motivations for our experiments.

Because competitive trading of a limited supply of permits is expected to distribute individual emissions choices efficiently despite imperfect enforcement, the main theoretical consequence of imperfect enforcement is that aggregate emissions will exceed the aggregate supply of emissions permits. Toward characterizing the equilibrium violations of noncompliant risk neutral firms, [3] indicates that a noncompliant firm chooses its violation to satisfy $p=\pi f^{\prime}\left(v_{i}\right)$. Note that a firm's violations depend on the permit price and the enforcement strategy all firms face, but it does not depend on anything that is unique about the firm. ${ }^{4}$ Since the determinants of the firms' violation choices are the same, if they are all noncompliant they all choose the same level of violation. What drives this result is the ability of a permit market to

\footnotetext{
${ }^{4}$ For this reason, Stranlund and Dhanda (1999) argued that a budget-constrained regulator that seeks to minimize the aggregate violations of heterogeneous risk neutral firms cannot use differences in the firms' control costs or initial permit allocations to target its monitoring effort. The reason is that each firm's emissions and violation decisions are keyed to the permit price so that, at the margin, there are no differences among them that an enforcer can exploit. Murphy and Stranlund (2007) use laboratory experiments to test and confirm the hypothesis that firms' violations choices are independent of their abatement costs. They do find, however, some evidence that violations may depend in part on whether firms are net buyers or net sellers of permits.
} 
equate the marginal incentives of risk-neutral firms to release emissions and to violate their permits.

Just like equating the firms’ marginal incentives to pollute maximizes aggregate gross profit given aggregate emissions, equating the marginal violation incentives of risk neutral firms minimizes aggregate expected penalties given aggregate emissions. Given $Q$, and a supply of permits $L$, aggregate violations are $V=Q-L$. Obviously we must have $V \geq 0$. It is straightforward to show that in a permit equilibrium,

$$
p=P^{\prime}(V) \text {, where } P(V)=\min _{v_{i}} \sum_{i=1}^{n} \pi f\left(v_{i}\right) \text { s.t. } \sum_{i=1}^{n} v_{i}=V \text {. }
$$

That is, $P(V)$ is minimum aggregate expected penalties for aggregate violations $V$, and in equilibrium the permit price is equal to the marginal of this function.

Having characterized the emissions and violation choices of risk neutral firms in a competitive emissions trading program, as well as their aggregate gross profits and expected penalties, let us now turn to the market equilibrium of such a program. Since the focus of this paper is on the industry-level welfare consequences of imperfect enforcement and compliance, we determine an equilibrium permit price and aggregate emissions, given an initial distribution of $L$ emissions permits and an enforcement strategy that cannot produce perfect compliance. Our results in [5] and [6] reveal a three-way equality that uniquely identifies the equilibrium permit price and aggregate emissions:

$$
p=B^{\prime}(Q)=P^{\prime}(Q-L)
$$

The equilibrium comparative statics of this problem are easy to demonstrate (see Stranlund and Dhanda 1999). Aggregate emissions and violations are increasing as enforcement is weakened, either by reducing the monitoring probability or reducing the marginal penalty function. Because weaker enforcement decreases the aggregate demand for permits, the 
equilibrium permit price falls. Increasing the supply of permits decreases the equilibrium permit price and increases aggregate emissions, but aggregate violations fall.

In this paper, however, we focus on the deviations of behavior in the lab from the predictions of the model described above. Moreover, since we are interested consequences of these deviations on industry-level payoffs, we will focus on the aggregates that our experimental markets produce — aggregate emissions, aggregate violations, aggregate gross profits, aggregate expected penalties, and aggregate expected net profits. Denote our actual observations of these values as $Q^{a}, V^{a}, B^{a}, P^{a}$, and $W^{a}$, respectively. Given our experimental design, the theory described above for risk-neutral profit-maximizing firms provides predictions for aggregate emissions and violations, $Q^{p}$ and $V^{p}$. Moreover, the theory predicts that the distribution of individual emissions will maximize aggregate gross profits, and the distribution of individual violations will minimize aggregate expected penalties. Thus, our prediction of aggregate gross profits is $B\left(Q^{p}\right)$ defined by [5], and our prediction of aggregate expected penalties is $P\left(V^{p}\right)$ defined by [6]. Finally, it is easy to demonstrate that the predictions of individual emissions and violations maximizes aggregate expected net profits, $W\left(Q^{p}\right)=B\left(Q^{p}\right)-P\left(V^{p}\right)$. (We write expected net profits as a function of aggregate emissions alone because these automatically determine aggregate violations given the supply of permits).

Empirically, observed deviations from model predictions can be decomposed into two effects: individual allocation effects and aggregate compliance effects. Individual allocation effects occur when individual emission and violation choices deviate from model predictions given the observed level of aggregate emissions and violations. Aggregate compliance effects arise whenever the observed level of aggregate emissions, and hence aggregate violations, deviate from the predictions. 
This decomposition of the deviations in observed and predicted aggregate gross profits is

$$
B^{a}-B\left(Q^{p}\right)=\underbrace{\left[B^{a}-B\left(Q^{a}\right)\right]}_{\text {Allocation Effect }}+\underbrace{\left[B\left(Q^{a}\right)-B\left(Q^{p}\right)\right]}_{\text {Compliance Effect }} .
$$

The individual allocation effect in [8] is the difference between observed and maximum aggregate gross profits, conditioned on the observed level of aggregate emissions. Recall that Malik (1992) showed that a competitive emissions permit market should allocate individual emissions to maximize aggregate gross profit at the observed level of aggregate emissions, regardless of firms’ preferences for risk. By definition, the allocation effect in [8] must be nonpositive, and if it is negative then the permit market has failed to distribute individual emissions efficiently.

Note that the aggregate compliance effect of deviations from predicted aggregate gross profits in [8] is conditioned on the assumption that the permit market distributes individual emissions efficiently. This term can be any sign depending on whether observed aggregate emissions ( $Q^{a}$ ) are greater than, less than, or equal to the theoretically predicted level, $Q^{p}$. Note that deviations in $B\left(Q^{a}\right)$ and $B\left(Q^{p}\right)$ do not indicate market failures because deviations of $Q^{a}$ from $Q^{p}$ could be due to differences in compliance incentives from those of risk-neutral profit-maximizing firms. Thus, if $B\left(Q^{a}\right)$ and $B\left(Q^{p}\right)$ differ, the failure is because the model failed to predict aggregate emissions correctly.

In the same way we can decompose the deviation between observed and predicted expected aggregate penalties:

$$
P^{a}-P\left(V^{p}\right)=\underbrace{\left[P^{a}-P\left(V^{a}\right)\right]}_{\text {Allocation Effect }}+\underbrace{\left[P\left(V^{a}\right)-P\left(V^{p}\right)\right]}_{\text {Compliance Effect }} .
$$


The allocation effect in this case is the difference between observed and minimum expected aggregate penalties, conditioned on observed aggregate violations. This term is non-negative, because $P\left(V^{a}\right)$ is minimum aggregate expected penalties given observed aggregate emissions $Q^{a}$, and hence, observed aggregate violations $V^{a}$. Again, deviations in observed and predicted expected penalties are not market failures. They indicate instead a failure of the model of riskneutral profit-maximizing firms to correctly predict individual violations. The compliance effect is conditioned on the firms choosing violations that minimize aggregate expected penalties, but will differ from zero if observed and predicted aggregate violations differ.

Finally, the allocation and compliance effects of deviations in aggregate expected net profits is

$$
W^{a}-W\left(Q^{p}\right)=\underbrace{\left[W^{a}-W\left(Q^{a}\right)\right]}_{\text {Allocation Effect }}+\underbrace{\left[W\left(Q^{a}\right)-W\left(Q^{p}\right)\right]}_{\text {Compliance Effect }} .
$$

The allocation effect on expected net profit is simply the allocation effect on gross profits in [8] minus the allocation effect on expected penalties in [9]. The compliance effect in [10] is defined in the same way.

\section{Experimental Design and Procedures}

\subsection{Experiment design}

Our experiments were designed to compare laboratory behavior to the predictions generated from the theory just described, but the subjects were placed in a more neutral environment. To avoid introducing potential biases due to individual attitudes about the environment or emissions trading, we framed the experiments as a production decision in which permits conveyed a license to produce, rather than an emissions decision. We also avoided the term "profit", choosing 
instead the more neutral term "benefit". (Throughout the paper, however, we use profits and emissions). We are mainly interested in whether aggregate gross profits, expected penalties and expected net profits differ from predicted values, and if so, the extent to which these deviations are due to the inability of a permit market to allocate individual emissions choices efficiently or to compliance choices by the subjects that differ from those of a profit-maximizing risk neutral individual. <INSERT TABLE 1>.

Table 1 summarizes the experimental design. During each period, subjects simultaneously chose to emit units of an unspecified pollutant and traded in a market for permits that conveyed the right to emit. In the nine Imperfect Enforcement treatments, subjects could emit as much as they wished (up to a capacity constraint) regardless of the number of permits, $l$, they owned. However, at the end of the period, each individual was audited with a known, exogenous probability. If an individual was audited and found to be non-compliant (i.e., total emissions exceeded permit holdings), then a penalty was applied. In the two Perfect Enforcement treatments, subjects were only able to trade in the permit market; emissions were automatically set equal to the final permit balance. Because there was no compliance choice in these two baseline treatments, there was, of course, no need for fines or audits.

Subjects received a gross profit from their choice of emissions, $q$, which was generated from a linear marginal gross profit function, $b^{\prime}(q)=18-\theta q$. Each experiment had eight subjects divided evenly into two types; subjects were randomly assigned a type. Subjects with a high marginal gross profit function $\left(\theta_{H}=1\right)$ also had a greater emission capacity of 17 units. Subjects with a low marginal gross profit function $\left(\theta_{L}=2\right)$ could emit up to 8 units. Emissions were constrained to be a whole number. 
Limiting the total supply of permits imposed an aggregate emissions standard, $L$. We consider two aggregate standards. In the experiments with a low aggregate standard ( $L_{L}=28$ ), denoted Low Standard, each of the four high marginal gross profit subjects was initially allocated $l^{0}=3$ permits, and the four low marginal gross profit subjects were each given four permits. In the high aggregate standard experiments $\left(L_{H}=56\right)$, denoted High Standard, there were two different initial allocations of permits. With the Uniform initial allocation, each of the eight subjects started with seven permits. With the Non-uniform initial allocation, the high marginal gross profit subjects began with $l^{0}=13$ permits, and the low marginal gross profit subjects had a single permit.

The treatment variables were the probability of an audit and the marginal penalty function (which together define an enforcement strategy), the initial permit allocation, and the total supply of permits. Each of the eleven treatments was repeated three times. In the Imperfect Enforcement treatments, each subject's record was examined with a known probability $\pi$. If a subject was found to be non-compliant, that is $q>l$, then she was assessed a penalty that was generated from a linearly increasing marginal penalty function, $f^{\prime}(q-l)=F+\phi(q-l)$. By changing the parameters of the expected marginal penalty function, $\pi f^{\prime}(q-l)=\pi[F+\phi(q-l)]$, we developed three enforcement strategies, which we label $\operatorname{Medium}\left(\pi_{H}\right)$, Medium $\left(\pi_{L}\right)$, and $\operatorname{Low}$. In theory, all risk neutral subjects should choose to be noncompliant under each of these levels of enforcement. The treatments Medium $\left(\pi_{H}\right)$ and Medium $\left(\pi_{L}\right)$ involved the same expected marginal penalties, but $\operatorname{Medium}\left(\pi_{H}\right)$ had a higher monitoring probability and a relatively low marginal penalty function ( $\pi_{H}=0.70, F=6, \phi=1.43$ ), whereas $\operatorname{Medium}\left(\pi_{L}\right)$ had a lower monitoring probability and a higher marginal penalty function $\left(\pi_{L}=0.35, F=12, \phi=2.90\right)$. Our intention here was to examine whether the subjects reacted differently to monitoring and penalties. The 
Low marginal expected penalty treatments had the weakest enforcement strategy with the low monitoring probability and a low marginal penalty function ( $\left.\pi_{L}=0.35, F=2, \phi=2.90\right)$. Enforcement parameter values were chosen, in part, so that the expected marginal penalty functions are parallel to each other—each has a slope of about one.

\subsection{Experiment procedures}

Participants were recruited from the student population at the University of Massachusetts, Amherst. Subjects were paid $\$ 7$ for agreeing to participate and showing up on time, and were then given an opportunity to earn additional money in the experiment. These additional earnings ranged between $\$ 5.68$ and $\$ 17.49$, with a mean of $\$ 13.67(\sigma=1.46)$. Earnings were paid in cash at the end of each experiment. Each experiment lasted about 2 hours.

The experiments were run in a computer lab using software designed specifically for this research. To familiarize subjects with the experiments, we ran a series of training experiments. In the first stage of the trainers, students read online instructions that included interactive questions to ensure that they understood the instructions before proceeding. After everyone had completed the instructions and all questions were answered, the training experiment began. These practice rounds contained all the same features as the "real data" experiments with the exception that we used a different set of parameters. The data from the trainers were discarded. We recruited participants from the pool of trained subjects for the real data sessions.

Subjects were allowed to participate in multiple sessions. A total of 176 subjects participated in 33 eight-person market experiments (11 treatments, three groups per treatment). Because subjects were allowed to participate in multiple sessions, the number of unique participants is less than the total required. Prior to the start of the real data experiments, subjects 
were given a summary of the experiment instructions. ${ }^{5}$ The experimenter read these instructions aloud and answered any questions. Each subject was given a calculator, a pencil and paper. Each experiment consisted of 12 identical rounds. At the start of each period, the eight subjects were each given an initial allocation of permits and \$10 in experimental cash.

A unique feature of our experiments is that the emission decisions and permit market trading were unbundled into two separate, but simultaneous, activities. We did this to allow for the possibility that the emission levels and permit holdings could differ, thereby introducing a compliance decision. During the period and concurrent with the production decision, subjects were able to alter their permit holdings by trading in a continuous double auction. In the auction, individuals could submit bids to buy or asks to sell a single permit. The highest bid and lowest ask price were displayed on the screen. A trade occurred whenever a buyer accepted the current ask or a seller accepted the current bid. After each trade, the current bid and ask were cleared and the market opened for a new set of bids and asks. The trading price history was displayed on the screen.

Each period lasted a total of five minutes. The permit market was open for the entire period, but production had to be completed in the first four minutes. The one-minute reconciliation period gave subjects a final opportunity to adjust their permit holdings. After each period ended, random audits were conducted and penalties were assessed. All information relating to audit outcomes was private.

\footnotetext{
5 The complete instructions, along with a summary are available online at http://faculty.cbpp.uaa.alaska.edu/jmurphy/research.html.
} 


\section{Results}

In this section we analyze the data from our experiments to investigate the effects of imperfect enforcement on aggregate gross profits, expected penalties and expected net profits. We are particularly interested in how our observations of these values differ from the predictions of the theoretical model of section. Our data is made up of 11 treatments (indexed by $k$ ), each of which was played by three groups (indexed by $j$ ) over 12 rounds (indexed by $t$ ). We are interested only in aggregate outcomes, not individual choices. The first two periods are omitted from the analysis to minimize the effects of learning, leaving 10 observations per group. (Omitting the first two rounds does not change any of our qualitative results). Therefore, we have 330 grouplevel observations (11 treatments, 3 groups per treatment, 10 rounds per group).

\subsection{The compliance and allocation effects of deviations from predicted gross profits}

We begin our analysis with the comparisons of aggregate emissions and violations (Table 2). For each treatment $k$, we compare the mean of observed aggregate emissions and violations (averaged over the 3 groups and 10 rounds of the treatment), denoted $Q^{a}$ and $V^{a}$, to the predictions, $Q^{p}$ and $V^{p}$, from the model of section 2. For the Imperfect Enforcement treatments, note that mean aggregate violations, $V^{a}$, follow the basic comparative static predictions of the model: violations tend to increase with weaker enforcement (given an aggregate standard, the Low enforcement strategy treatment has the most violations) and lower aggregate standard (given an enforcement strategy, violations are higher under the Low Standard).

The last column of Table 2 presents the differences between mean and predicted aggregate violations. Since each treatment has a fixed supply of permits, this difference in aggregate violations is identical to the difference in aggregate emissions; that is, 
$V^{a}-V^{p} \equiv Q^{a}-Q^{p}$. To investigate the statistical significance of these differences we employ the following mixed effects linear regression:

$$
V_{k j t}^{a}-V_{k}^{p}=\alpha+\sum_{k=1}^{8} \beta_{k} X_{k j t}+v_{j}+\varepsilon_{k j t},
$$

where $X$ is the set of dummy variables for eight of the nine imperfect compliance treatments, with Low Standard, Medium $\left(\pi_{H}\right)$ Enforcement as the omitted dummy variable. ${ }^{6}$ For the Perfect Enforcement treatments, predicted and mean aggregate violations (and aggregate emissions) are zero by design and are therefore not included in the regression model.

The most important results from Table 2 will have impacts throughout the analysis: the differences between mean and predicted aggregate violations, $V^{a}-V^{p}$, are negative and statistically significant for the three Low Standard treatments, but are statistically indistinguishable from zero for the six High Standard treatments. This suggests that individual choose lower-than-predicted violations when they are predicted to choose high violations, but that there violations are close to predicted values when these predictions are low. Clearly, the standard theoretical model of compliance in emissions trading programs does not accurately predict aggregate violations when enforcement is very weak. <INSERT TABLE 2>

\subsection{The compliance and allocation effects of deviations from predicted gross profits}

We now turn to analyzing aggregate gross profits using the results shown in Table 3 . If the emissions permit market is perfectly competitive and all firms are profit maximizers, then for each treatment, the predicted aggregate gross profit is $B\left(Q^{p}\right)$ from [5]. That is, $B\left(Q^{p}\right)$ is

\footnotetext{
${ }^{6}$ For each treatment $k$, Table 2 reports the results of a Wald $\chi^{2}$ test of the null hypothesis that $\alpha+\beta_{k}=0$, or in the case of the omitted dummy variable, Low Standard, Medium $\left(\pi_{H}\right)$ Enforcement, $\alpha=0$.
} 
maximum aggregate gross profit achievable given predicted aggregate emissions $Q^{p}$. For each treatment, the average (over groups and rounds) of observed aggregate gross profits is denoted $B^{a}$. In order to decompose the difference between observed and predicted aggregate gross profits into allocation and compliance effects, we calculated the maximum possible gross profit given the observations of aggregate emissions for each group, round, and treatment. In Table 3 we report the means of these values for each treatment, $B\left(Q^{a}\right) .<$ INSERT TABLE $3>$

The results in the $B^{a}-B\left(Q^{p}\right)$ column of Table 3 show that observed aggregate gross profits are essentially equal to predicted gross profits under the Perfect Enforcement treatments. This simply replicates the well-known result that standard double auctions with perfect compliance yield highly efficient outcomes. However, under the Imperfect Enforcement/Low Standard treatments, observed gross profits are significantly lower than predicted gross profits. Under the Imperfect Enforcement/High Standard treatments, the deviations of mean observed gross profits from predictions are not statistically different for all but two of six cases. In percentage terms, $B^{a} / B\left(Q^{p}\right)$, observed gross profits are always more than $97 \%$ of predicted gross profits under the Imperfect Enforcement/High Standard, while they range from only 80\% to $94 \%$ of predicted gross profits under the Imperfect Enforcement/Low Standard treatments.

But recall that a deviation of observed gross profits from the predicted value does not necessarily indicate that the permit market fails to distribute individual emission control efficiently. Such a deviation could be due differences in observed and predicted aggregate emissions that come from differences between actual and risk-neutral profit maximizing compliance choices. To better explain the differences between observed and predicted gross profits, we decomposed these differences into the allocation and compliance effects in equation 
[8] for each group in each round and present the means of these values for each treatment in Table 3.

The allocation effects in Table 3 provide a direct test of Malik's (1990) hypothesis that permit markets will allocate individual emissions efficiently despite imperfect enforcement. If this hypothesis were to hold, then all the allocation effects in Table 3 would be statistically indistinguishable from zero; clearly they are not. Note that the allocation effects are essentially zero in our Perfect Enforcement treatments. Thus, when we did not give the subjects the opportunity to violate their permits, they successfully allocated emissions among themselves to maximize aggregate gross profits. However, the allocation effects are statistically significant in all nine Imperfect Enforcement treatments. From a practical perspective, however, these effects are very small and not economically significant since mean observed aggregate gross profits, $B^{a}$, are always at least $96 \%$ of the maximum possible value given the observed level of emissions, $B\left(Q^{a}\right)$. We therefore conclude that the permit markets allocated individual emissions choices efficiently, despite imperfect enforcement, significant noncompliance, and emissions and violation choices that sometimes differ from their predicted values.

In light of the previous discussion about the differences between observed and predicted aggregate violations (the results in Table 2), the compliance effects in Table 3 are not surprising. By design, the individual compliance effect is exactly zero in our Perfect Enforcement treatments. For the Imperfect Enforcement treatments, the compliance effects are negative and significant only for the Low Standard treatments; these effects are statistically indistinguishable from zero in all of the High Standard treatments. These results are simply a translation into aggregate gross profits of the result that actual violations are significantly lower than predicted for the Low Standard treatments but are essentially zero in the High Standard treatments. 
The large compliance effects for the Imperfect Enforcement/Low Standard treatments is the reason that mean observed gross profits are significantly lower than predicted in these treatments. That is, the permit markets were reasonably efficient at allocating individual emissions, but the subjects chose significantly lower-than-predicted violations. Mean observed gross profits are very close to predicted values for the Imperfect Enforcement/High Standard treatments because the allocations effects are small and the compliance effects are indistinguishable from zero. Again, the markets were reasonably efficient, but in these treatments aggregate violations were essentially the same as predicted.

\subsection{The compliance and allocation effects of deviations from predicted expected penalties}

We now examine deviations in observed expected penalties from predicted values and decompose these deviations into allocation and compliance effects. The results are in Table 4. If the subjects are risk-neutral profit-maximizers, then for each treatment, the predicted aggregate expected penalties are $P\left(V^{p}\right)$ from [6]. That is, $P\left(V^{p}\right)$ is minimum aggregate expected penalties given predicted aggregate emissions $V^{p}$.

The means of observed expected penalties for each treatment, denoted $P^{a}$, are calculated as follows. For each individual in each round of the experiments, we calculated the penalty that she would incur if audited for her actual level of violations; this was then multiplied by the audit probability to get her expected penalty. These values were then summed over the individuals in a group in each round to obtain aggregate expected penalties for each group in each round. In Table 4 we present the means of these values (averaged over groups and rounds) for each treatment. Note that these are the averages of aggregate expected penalties, not the means of the penalties that were actually imposed as a result of the random audits. This is the appropriate 
metric as we are interested in the choices that were made given the ex ante expected penalties, not the ex post realizations. Obviously these values are equal to zero in the Perfect Enforcement treatments. It is also important to recall that differences between $P^{a}$ and $P\left(V^{p}\right)$ do not indicate permit market failures; they are due instead to differences in compliance choices from those of risk-neutral, profit-maximizing agents. Finally, to calculate the allocation and compliance effects of the differences between observed and predicted expected penalties, we calculated the minimum possible aggregate expected penalty given the observed aggregate violations for each group, round, and treatment. In Table 4 we report the means of these values for each treatment, $P\left(V^{a}\right) .<$ INSERT TABLE $4>$

Note that mean observed expected penalties are significantly lower than predicted for the three Imperfect Enforcement/ Low Standard treatments. In contrast, mean expected penalties are higher that predicted for the Imperfect Enforcement/ High Standard treatments, but the differences are statistically significant in only two of six cases. Note, however, that the allocations effects are always positive and significant (although weakly significant in one case). This indicates that, given the observed levels of aggregate violations, individual violations were not distributed in the way that would minimize aggregate expected penalties. The compliance effects are negative and significant for the Imperfect Enforcement/ Low Standard treatments while they are not different from zero in the Imperfect Enforcement/ High Standard treatments. These simply reflect that facts that the subjects chose significantly lower-than-predicted violations in the Imperfect Enforcement/ Low Standard treatments, while aggregate violations were very close to predicted values under the Imperfect Enforcement/ High Standard treatments. 


\subsection{The compliance and allocation effects of deviations from predicted expected net profits}

In this final section, we pull together our results about deviations in aggregate gross profits and expected penalties from predicted values to answer the basic question of how well the groups in our experiments performed relative to predictions. That is, we examine deviations in aggregate expected net profits (gross profits minus expected penalties) from predictions.

In Table 5 we first present predicted net profits, $W\left(Q^{p}\right)=B\left(Q^{p}\right)-P\left(V^{p}\right)$. Recall that $W\left(Q^{p}\right)$ is maximized expected net profit: the predicted levels of aggregate emissions and resulting aggregate violations, as well as the levels of individual emissions and violations, maximize the expected aggregate payoff to the industry. We also present mean observed expected net profits, $W^{a}=B^{a}-P^{a}$, and mean maximized expected net profits at the observed levels of aggregate emissions, $W\left(Q^{a}\right)=B\left(Q^{a}\right)-P\left(V^{a}\right)$. Note that the differences between predicted and mean observed expected net profits, $W^{a}-W\left(Q^{p}\right)$, is negative and significant for each treatment. However, they are very close to their maximized values. In percentage terms, observed expected net profits are very nearly 100\% of maxima for the Perfect Compliance Treatments; they exceed $95.8 \%$ of maxima for each of the Imperfect Enforcement/ High Standard treatments; they are somewhat lower for the Imperfect Enforcement/ Low Standard treatments, ranging from $87 \%$ to $96 \%$ of maximized expected net profits.

Mean observed expected net profits deviate from maximized values by greater amounts (in percentage terms) under the Imperfect Enforcement/ Low Standard treatments because of the significant compliance effects in these treatments. Although the allocation effects for all Imperfect Enforcement treatments are significant, they are similar in magnitudes across the Low Standard and High Standard treatments. However, the compliance effects in the Imperfect Enforcement/Low Standard treatments are large and significant, while they are very small and 
insignificant in the Imperfect Enforcement/ High Standard treatments. The groups under the Imperfect Enforcement/Low Standard treatments tended to do worse mainly because they chose significantly lower-than-predicted violations.

\section{Conclusion}

The most important results of our work are that experimental emissions permit markets are highly efficient at allocating individual emissions control, despite imperfect enforcement and significant violations, but aggregate violations may not be as high as one would predict with a standard model of behavior, particularly when the model predicts high levels of noncompliance. In treatments in which subjects did not violate their permits as much as predicted, mean aggregate expected payoffs were lower than maximized expected payoffs. It is important to reiterate that this finding does not imply some sort of inefficiency. Rather it appears to be due mainly to violation choices that differ from those that a risk-neutral payoff-maximizing individual would make. If there is a failure, it is a failure of the standard model to accurately predict compliance choices, not a failure of permit markets to allocate emissions efficiently.

In fact, lower-than-predicted violation choices can mean higher social welfare. We have focused our analysis on the welfare of firms operating under imperfectly enforced emissions trading, but presumably there are damages from their emissions. We have not said anything about pollution damage, because including it in our analysis can only be done in an ad hoc manner. Nevertheless, when violations are lower-than-predicted, pollution damage is lower-than-

predicted. It is easy to come up with specifications of damage functions for our experiments with which the lower-than-predicted violations we observe lead to higher-than-predicted social welfare. 
One should be careful about this possibility. It does not mean that imperfect enforcement of emissions trading programs is somehow desirable; it only means that imperfect enforcement may in some situations be less costly than standard models would predict. Recent theoretical developments suggest that it is likely to be efficient to design tradable rights programs that achieve perfect compliance (Stranlund 2007). Moreover, several current emissions trading schemes have achieved perfect, or at least near perfect, compliance (e.g. the EPA's $\mathrm{SO}_{2}$ and $\mathrm{NO}_{\mathrm{X}}$ Budget trading programs). Our analysis does not address these situations. Instead, our analysis is motivated by the fact that implementing emissions trading policies beyond their current applications will often mean moving them into contexts in which inducing and maintaining perfect compliance will be more difficult. With the continuing application of market-based policies into new regulatory settings, it is virtually certain that regulators will have to confront the problems associated with imperfect enforcement, if they have not had to do so already. Our analysis provides valuable information about the likely welfare effects of failing to induce perfect compliance with tradable property rights regulations. 


\section{References}

Alm, J. and M. McKee. 1998. "Extending the Lessons of Laboratory Experiments on Tax Compliance to Managerial and Decision Economics.” Managerial and Decision Economics 19(4-5), 259-275.

Cason, T. N. and L. Gangadharan. 2006. "Emissions Variability in Tradable Permit Markets with Imperfect Enforcement and Banking.” Journal of Economic Behavior and Organization 61(2), 199-216.

Isaac, R.M. and C. Holt. 1999. Research in Experimental Economics: Emissions Permit Experiments. Vol. 7. Stamford, CT: JAI Press, Inc.

Keeler, A. 1991. "Noncompliant Firms in Transferable Discharge Permit Markets: Some Extensions.” Journal of Environmental Economics and Management 21(2), 180-189.

Malik, A. S. 1990. "Markets for Pollution Control when Firms are Noncompliant.” Journal of Environmental Economics and Management 18(2), 97-106.

Malik, A. S. 1992. "Enforcement Cost and the Choice of Policy Instruments for Controlling Pollution.” Economic Inquiry 30(4), 714-721.

Montgomery, W. D. 1972. "Markets in Licenses and Efficient Pollution Control Programs." Journal of Economic Theory 5(3), 395-418.

Muller, R.A. and S. Mestelman. 1998. "What Have We Learned from Emissions Trading Experiments?” Managerial and Decision Economics. 19(4-5):225-238.

Murphy, J. J. and J. K. Stranlund. 2006. "Direct and Indirect Effects of Enforcing Emissions Trading Programs: An Experimental Analysis.” Journal of Economic Behavior and Organization 61(2), 217-233.

Murphy, J. J. and J. K. Stranlund. 2007. “A Laboratory Investigation of Compliance Behavior under Tradable Emissions Rights: Implications for Targeted Enforcement.” Journal of Environmental Economics and Management 53(2), 196-212.

Stranlund, J. K. and K. K. Dhanda. 1999. "Endogenous Monitoring and Enforcement of a Transferable Emissions Permit System.” Journal of Environmental Economics and Management 38(3), 267-282.

Stranlund, J. K. 2007. "The Regulatory Choice of Noncompliance in Emissions Trading Programs.” Environmental and Resource Economics 38(1), 99-117.

Torgler, B. 2002. "Speaking to Theorists and Searching for Facts: Tax Morale and Tax Compliance in Experiments.” Journal of Economic Surveys 16(5), 657-683.

U.S. Environmental Protection Agency. 2007a. Acid Rain and Related Programs: 2006 Compliance Report. http://www.epa.gov/airmarkets/progress/docs/2006-ARP-Report.pdf. Last access: March 20, 2008.

U.S. Environmental Protection Agency. 2007b. $N O_{X}$ Budget Trading Program: 2006 Program Compliance and Environmental Results. http://www.epa.gov/airmarkets/progress/docs/2006NBP-Report.pdf. Last access: March 20, 2008. 


\section{Table 1. Experimental Design}

\section{Enforcement Aggregate Standard / Initial Allocation}

\begin{tabular}{|c|c|}
\hline \multicolumn{2}{|c|}{ Perfect Enforcement Treatments } \\
\hline (none) & $\begin{array}{l}\text { Low Standard/ Uniform } \\
\text { High Standard/ Uniform }\end{array}$ \\
\hline \multicolumn{2}{|c|}{ Imperfect Enforcement Treatments } \\
\hline $\operatorname{Medium}\left(\pi_{H}\right)$ & $\begin{array}{l}\text { Low Standard /Uniform } \\
\text { High Standard/ Uniform } \\
\text { High Standard / Non-uniform }\end{array}$ \\
\hline $\operatorname{Medium}\left(\pi_{L}\right)$ & $\begin{array}{l}\text { Low Standard / Uniform } \\
\text { High Standard / Uniform } \\
\text { High Standard /Non-uniform }\end{array}$ \\
\hline Low & $\begin{array}{l}\text { Low Standard / Uniform } \\
\text { High Standard Uniform } \\
\text { High Standard / Non-uniform }\end{array}$ \\
\hline
\end{tabular}

Each treatment was repeated three times with eight participants per group. 
Table 2. Predicted and Actual Aggregate Emissions and Violations

Treatment

Low Standard

Perfect Enforcement

Medium $\left(\pi_{H}\right)$

Medium $\left(\pi_{L}\right)$

Low

$$
Q^{p} \quad Q^{a} \quad V^{p} \quad V^{a} \quad V^{a}-V^{p}
$$

$\begin{array}{ccccc}28 & 28 & 0 & & \\ 52 & 48.4 & 24 & 20.4 & -3.6^{* * *} \\ 52 & 40.4 & 24 & 12.4 & -11.6^{* * *} \\ 64 & 55.7 & 36 & 27.7 & -8.3^{* * *}\end{array}$

High Standard Uniform Allocation

Perfect Enforcement

$\begin{array}{ccccr}56 & 56 & 0 & & \\ 64 & 63.5 & 8 & 7.5 & -0.5 \\ 64 & 65.4 & 8 & 8.9 & 0.9 \\ 76 & 76.3 & 20 & 20.3 & 0.3\end{array}$

High Standard Non-uniform Allocation

\section{Perfect Enforcement}

$\begin{array}{llllll}\text { Medium }\left(\pi_{H}\right) & 64 & 63.9 & 8 & 7.9 & -0.1\end{array}$

$\begin{array}{llllll}\text { Medium }\left(\pi_{L}\right) & 64 & 65.4 & 8 & 9.4 & 1.4\end{array}$

Low $\quad \begin{array}{rrrrr}76 & 74.5 & 20 & 18.5 & -1.5\end{array}$

Significance levels $\stackrel{*}{p}<0.10$, $* * p<0.05$; *** $p<0.01$, are for tests of the null hypothesis that the value is equal to zero. Wald $\chi^{2}$ tests are obtained from a linear regression of $V^{a}-V^{p}$ with dummy variables for each treatment as the fixed effects and allowing for random effects across groups and periods. 
Table 3. The Compliance and Allocation Effects of Deviations from Predicted Gross Profits

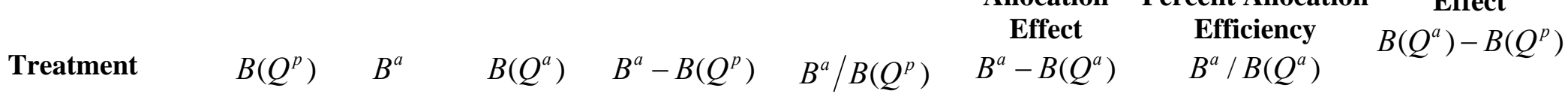

Low Standard

$\begin{array}{lccccccrr}\text { Perfect Enforcement } & 420 & 417.1 & 420 & -2.9 & 99.3 \% & -2.9 & 99.3 \% & 0 \\ \text { Medium }\left(\boldsymbol{\pi}_{\boldsymbol{H}}\right) & 676 & 634.1 & 642.4 & -41.9^{* * *} & 93.8 \% & -8.3^{* * *} & 98.7 \% & -33.6^{* * *} \\ \text { Medium }\left(\boldsymbol{\pi}_{\boldsymbol{L}}\right) & 676 & 542.5 & 560.9 & -133.5^{* * *} & 80.2 \% & -18.5^{* * *} & 96.7 \% & -115.1^{* * *} \\ \text { Low } & 768 & 692.8 & 705.3 & -75.2^{* * *} & 90.2 \% & -12.5^{* * *} & 98.2 \% & -62.7^{* * *}\end{array}$

High Standard Uniform Allocation

\begin{tabular}{|c|c|c|c|c|c|c|c|c|}
\hline Perfect Enforcement & 708 & 706.6 & 708 & $-1.4^{*}$ & $99.8 \%$ & $-1.4^{*}$ & $99.8 \%$ & 0 \\
\hline $\operatorname{Medium}\left(\pi_{H}\right)$ & 768 & 758.3 & 763.6 & -9.7 & $98.7 \%$ & $-5.3^{* *}$ & $99.3 \%$ & -4.4 \\
\hline $\operatorname{Medium}\left(\pi_{L}\right)$ & 768 & 766.9 & 772.7 & -1.1 & $99.9 \%$ & $-5.8^{* *}$ & $99.3 \%$ & 4.7 \\
\hline Low & 836 & 823.8 & 836.2 & -12.2 & $98.5 \%$ & $-12.4^{* * *}$ & $98.5 \%$ & 0.2 \\
\hline \multicolumn{9}{|c|}{ High Standard Non-uniform Allocation } \\
\hline $\operatorname{Medium}\left(\pi_{H}\right)$ & 768 & 751.0 & 766.0 & $-17.0^{* *}$ & $97.8 \%$ & $-15.0^{* * *}$ & $98.1 \%$ & -2.0 \\
\hline $\operatorname{Medium}\left(\pi_{L}\right)$ & 768 & 768.4 & 776.1 & 0.4 & $100.0 \%$ & $-7.7^{* * *}$ & $99.0 \%$ & 8.1 \\
\hline Low & 836 & 816.9 & 827.5 & $-19.1^{* *}$ & $97.7 \%$ & $-10.6^{* * *}$ & $98.7 \%$ & -8.5 \\
\hline
\end{tabular}

Significance levels ${ }^{*} p<0.10, * * p<0.05$, and $* * * p<0.01$, are for tests of the null hypothesis that the value is equal to zero. Wald $\chi^{2}$ tests are obtained from linear regressions of each variable with dummies for each treatment as the fixed effects for treatment and allowing for random effects across group and period. 
Table 4. The Compliance and Allocation Effects of Deviations from Predicted Expected Penalties

$\begin{array}{llllllll} & & & \begin{array}{c}\text { Allocation } \\ \text { Effect }\end{array} & \begin{array}{c}\text { Compliance } \\ \text { Effect }\end{array} \\ \text { Treatment } & P\left(V^{p}\right) & P^{a} & P\left(V^{a}\right) & P^{a}-P\left(V^{p}\right) & P^{a} / P\left(V^{p}\right) & P^{a}-P\left(V^{a}\right) & P\left(V^{a}\right)-P\left(V^{p}\right)\end{array}$

Low Standard

\begin{tabular}{|c|c|c|c|c|c|c|c|}
\hline Perfect Enforcement & 0 & 0 & 0 & & & & \\
\hline $\operatorname{Medium}\left(\pi_{H}\right)$ & 148.9 & 129.0 & 123.2 & $-19.9^{* * *}$ & $86.6 \%$ & $5.8^{* * *}$ & $-25.7^{* * *}$ \\
\hline $\operatorname{Medium}\left(\pi_{L}\right)$ & 149.5 & 81.1 & 70.4 & $-68.4^{* * *}$ & $54.3 \%$ & $10.8^{* *}$ & $-79.4^{* * *}$ \\
\hline Low & 126.7 & 94.9 & 83.9 & $-31.8^{* * *}$ & $74.9 \%$ & $11.1^{* * *}$ & $-42.8^{* * *}$ \\
\hline
\end{tabular}

High Standard Uniform Allocation

$\begin{array}{lccccccc}\text { Perfect Enforcement } & 0 & 0 & 0 & & & & \\ \text { Medium }\left(\boldsymbol{\pi}_{\boldsymbol{H}}\right) & 41.6 & 45.1 & 40.2 & 3.5 & 108.5 \% & 5.0^{*} & -1.4 \\ \text { Medium }\left(\boldsymbol{\pi}_{\boldsymbol{L}}\right) & 41.7 & 55.5 & 48.1 & 13.8 & 133.0 \% & 7.4^{* *} & 6.4 \\ \text { Low } & 50.5 & 71.4 & 51.9 & 20.9^{* *} & 141.3 \% & 19.6^{* * *} & 1.3\end{array}$

High Standard Non-uniform Allocation

$\begin{array}{lllllllc}\text { Medium }\left(\boldsymbol{\pi}_{\boldsymbol{H}}\right) & 41.6 & 48.0 & 41.9 & 6.4 & 115.3 \% & 6.1^{* *} & 0.4 \\ \text { Medium }\left(\boldsymbol{\pi}_{\boldsymbol{L}}\right) & 41.7 & 68.3 & 51.1 & 26.5^{* *} & 163.6 \% & 17.1^{* * *} & 9.4 \\ \text { Low } & 50.5 & 62.8 & 45.5 & 12.2 & 124.2 \% & 17.3^{* *} & -5.1\end{array}$

Significance levels ${ }^{*} p<0.10, * * p<0.05 ; * * * p<0.01$, are for tests of the null hypothesis that the value is equal to zero. Wald $\chi^{2}$ tests are obtained from a linear regression with dummy variables for each treatment as the fixed effects and allowing for random effects across groups and periods. 
Table 5. The Compliance and Allocation Effects of Deviations from Predicted Expected Net Profits

$$
\begin{aligned}
& \text { Allocation Compliance } \\
& \text { Effect Effect } \\
& \text { Treatment } \quad W\left(Q^{p}\right) \quad W^{a} \quad W\left(Q^{a}\right) \quad W^{a}-W\left(Q^{p}\right) \quad W^{a} / W\left(Q^{p}\right) \quad W^{a}-W\left(Q^{a}\right) W\left(Q^{a}\right)-W\left(Q^{p}\right)
\end{aligned}
$$

Low Standard

$\begin{array}{lccccccc}\text { Perfect Enforcement } & 420 & 417.1 & 420 & -2.9^{*} & 99.3 \% & -2.9^{*} & 0 \\ \text { Medium }\left(\boldsymbol{\pi}_{\boldsymbol{H}}\right) & 527.2 & 505.1 & 519.2 & -22.0^{* * *} & 95.8 \% & -14.1^{* * *} & -7.9^{* * *} \\ \text { Medium }\left(\boldsymbol{\pi}_{\boldsymbol{L}}\right) & 526.5 & 461.3 & 490.6 & -65.1^{* * *} & 87.6 \% & -29.2^{* * *} & -35.9^{* * *} \\ \text { Low } & 641.3 & 597.8 & 621.4 & -43.5^{* * *} & 93.2 \% & -23.6^{* * *} & -19.9^{* * *}\end{array}$

High Standard Uniform Allocation

$\begin{array}{lccccccc}\text { Perfect Enforcement } & 708 & 706.6 & 708 & -1.4^{* * *} & 99.8 \% & -1.4^{* *} & 0 \\ \text { Medium }\left(\boldsymbol{\pi}_{\boldsymbol{H}}\right) & 726.4 & 713.2 & 723.5 & -13.2^{* * *} & 98.2 \% & -10.3^{* *} & -2.9 \\ \text { Medium }\left(\boldsymbol{\pi}_{\boldsymbol{L}}\right) & 726.3 & 711.4 & 724.6 & -14.9^{* * *} & 98.0 \% & -13.2^{* * *} & -1.7 \\ \text { Low } & 785.5 & 752.4 & 784.3 & -33.1^{* * *} & 95.8 \% & -32.0^{* * *} & -1.2\end{array}$

High Standard Non-uniform Allocation

$\begin{array}{llllllll}\text { Medium }\left(\boldsymbol{\pi}_{\boldsymbol{H}}\right) & 726.4 & 703.0 & 724.1 & -23.4^{* * *} & 96.8 \% & -21.1^{* * *} & -2.3 \\ \text { Medium }\left(\boldsymbol{\pi}_{\boldsymbol{L}}\right) & 726.3 & 700.2 & 725.0 & -26.1^{* * *} & 96.4 \% & -24.8^{* * *} & -1.3 \\ \text { Low } & 785.5 & 754.1 & 782.1 & -31.3^{* * *} & 96.0 \% & -27.9^{* * *} & -3.4\end{array}$

Significance levels ${ }^{*} p<0.10, * * p<0.05 ; * * * p<0.01$, are for tests of the null hypothesis that the value is equal to zero. Wald $\chi^{2}$ tests are obtained from a linear regression with dummy variables for each treatment as the fixed effects and allowing for random effects across groups and periods. 